\title{
LINEAR ASSESSMENT OF CEREBRAL AUTOREGULATION IN IDIOPATHIC PARKINSON'S DISEASE
}

\author{
CHUANG-CHIEN CHIU1,2*, KEN YING-KAI LIAO², SHOOU-JENG YEH²,3 \\ 1,2Department of Automatic Control Engineering, Feng Chia University, Taichung, Taiwan, R. 0. C., ${ }^{3 S e c t i o n ~ o f ~ N e u r o l o g y ~ a n d ~}$ \\ Neurophysiology, Cheng-Ching General Hospital, Taichung, Taiwan, R. O. C.
}

Email: chiuc@fcu.edu.tw

Received: 23 Apr 2016 Revised and Accepted: 29 May 2016

\begin{abstract}
Parkinson's disease cases have been on the rise in the recent years, which promoted several different researches into the disorder. However, there hasn't much research been done in the non-motor aspects of the disease. This study aims to improve the understanding of one of the non-motor symptoms of Parkinson's disease. Specifically, this research aims to further understand cerebral autoregulation in patients with Parkinson's disease. In order to achieve this aim, 25 subjects were recruited, with 11 healthy controls and 14 patients with Parkinson's disease. The continuous blood pressure and continuous cerebral blood flow velocity of all subjects were recorded and processed while the subjects were at rest, tilt-up, and during hyperventilation. Linear signal and system analysis techniques were applied such as the power spectral density analysis and cross-correlation function analysis. Results showed that patients with Parkinson's disease did not show a significant difference from the control group while at rest and after tilt-up. However, there was a significant difference between the groups during hyperventilation. The results obtained in this study suggested that the metabolic regulatory pathway for cerebral autoregulation is impaired in patients with Parkinson's disease.
\end{abstract}

Keywords: Cerebral autoregulation, Parkinson's disease, Hyperventilation

(c) 2016 The Authors. Published by Innovare Academic Sciences Pvt Ltd. This is an open access article under the CC BY license (http://creativecommons.org/licenses/by/4.0/) DOI: http://dx.doi.org/10.22159/ijpps.2016v8s2.15212.

\section{INTRODUCTION}

Parkinson's disease (PD) is a neurodegenerative disorder which is generally known to affect movement. It was first described by James Parkinson in 1817 as the shaking palsy. As medical advancements promoted life expectancy, the incidence rate of PD has also increased. In recent years, PD was not found only to affect the elderly, but young people as well, most notably the actor Michael J. Fox. With PD becoming more and more prevalent, more and more research has been poured into understanding the mechanisms of how PD works, as well as the motor symptoms of PD. However, it wasn't until recently that non-motor symptoms of PD became an interest to researchers [1]

Since PD is a neurodegenerative disease to the central nervous system, it is also of interest for researchers to investigate the autonomic nervous system (ANS) of PD patients. The investigation of ANS in PD patients have shown that ANS should be affected by PD as well [2]. It has also been shown that orthostatic hypotension $(\mathrm{OH})$ is present in patients with $\mathrm{PD}$ [3]. As it has been shown that $\mathrm{OH}$ is found in PD patients, it is then also important to ask whether or not cerebral autoregulation (CA) is impaired in PD. This is because in cases of $\mathrm{OH}$, it has been found that $\mathrm{CA}$ is not as effective, and this could prove to be dangerous for patients with PD [4].

In a recent study, it was found that there may be impairment of CA in PD since the recovery peak to baseline difference for cerebral blood flow velocity was lower than control subjects [5]. Also, in a recent $\mathrm{Ph}$. D. dissertation, the topic came up again and concluded that CA may not necessarily be impaired in PD patients, but it is altered [6]. Since the information in this subject is lacking, this research is dedicated to investigate further into CA and PD.

One interesting thing to consider when investigating $\mathrm{CA}$ is the fact that CA is affected largely by the concentration of carbon dioxide found in the body [7]. During hypercapnia, CA responds slower, and consequently, during hypocapnia, CA responds faster. In recent studies, the relationship between $\mathrm{CA}$ and carbon dioxide has been lightly touched upon $[8,9]$. This study aims further to investigate the relationship between CA and carbon dioxide in PD patients using techniques that had proven to worked to quantify CA with healthy controls as well as patients with diabetic autonomic neuropathy [10-12].

\section{MATERIALS AND METHODS}

\section{Subjects}

Patients with PD as well as age and gender-matched healthy controls were recruited for the purposes of this research, with permission granted by the investigation review board of Taichung Cheng-Ching General hospital, Taiwan. A total of 25 subjects were recruited for this study, including 11 healthy controls involving 8 males and 3 females with an average age of $56.5 \pm 8.6$ and 14 patients with PD including 8 males and 6 females with an average age of $58.3 \pm 12.5$ Patients were assessed by the clinical doctors using the Unified Parkinson's disease rating scale and were assessed to be not affected by or receiving treatment for any other disease or disorders. The subjects have also been instructed not to consume any drugs, alcohol, or caffeine three hours prior to the testing.

\section{Instruments}

The signals required by this study are continuous blood pressure signals and continuous cerebral blood flow velocity signals. The signals were acquired at Taichung Cheng-Ching General Hospital Autonomic Nervous System Testing Laboratory, using a Finapres (Ohmeda 2300 Finapres device; Finapres, Eaglewood, CO) device for the acquisition of continuous blood pressure signal by attaching the cuff on the right middle finger, and using a transcranial Doppler ultrasound (transcranial Doppler ultrasound, EME TC2020) for the acquisition of continuous cerebral blood flow velocity signal by using the transcranial Doppler ultrasound with a $5 \mathrm{MHz}$ transducer attached to an elastic headband fixed over the temporal bones. A custom program written in LabVIEW $®$ was used to record and digitize both signals and store into a personal computer.

\section{Experimental protocol}

The composite autonomic scoring scale experiment was used as the basis of the experimental design [13]. The subjects were instructed to rest for $10 \mathrm{~min}$ in the supine position (rest) while having their continuous blood pressure and continuous cerebral blood flow velocity recorded, then the subjects are passively tilted to 70 degrees using a tilting table, and the signals are recorded for a further $10 \mathrm{~min}$ (tilt), then the subjects were allowed to return to the supine position for a 10-minute break before undergoing 3 min of hyperventilation (HV). 


\section{Pre-processing}

Both peaks and valleys of the continuous blood pressure and continuous cerebral blood flow velocity signals were detected, and the mean of each waveform between the valleys were calculated in order to transform the signals into mean blood pressure (MBP) and mean cerebral blood flow velocity (MCBFV) of each heartbeat [10].

\section{Power spectral density}

For both the MBP and MCBFV signals, the power spectral density (PSD) is calculated in order to see the power distribution throughout the different frequency bands, which should reveal some information regarding the autonomic nervous system, based on the technique from heart rate variability. The mean is subtracted through an utterance-based cepstral mean subtraction in order to determine the mean removed signal $\widehat{\mathbf{x}}(\mathbf{n})$. This is shown in (1) below.

$$
\begin{array}{r}
\hat{x}(n)=x(n)-\mu_{x} \ldots \ldots \ldots \ldots \ldots \text { (1) } \\
\mu_{x}=\frac{1}{N} \sum_{n=1}^{N} x_{n}, n=1,2, \ldots, N \ldots \ldots \ldots \ldots \ldots
\end{array}
$$

Where $\mu_{\mathrm{x}}$ is the mean of the signal, determined through (2) above. The signal is transformed to the frequency domain through the use of Fast Fourier Transform, shown in (3) below, and the signal is transformed into the PSD, shown in (4) below. The powers of each frequency were then grouped into three ranges: The very low frequency (VLF) range which consisted of $0 \mathrm{~Hz}$ to $0.04 \mathrm{~Hz}$, the low frequency (LF) range which consisted of $0.04 \mathrm{~Hz}$ to $0.15 \mathrm{~Hz}$, and the high frequency (HF) range which consisted of $0.15 \mathrm{~Hz}$ to $0.4 \mathrm{~Hz}$.

$$
\begin{array}{r}
\hat{X}(k)=T \sum_{n=0}^{N-1} \hat{x}(n) e^{-j \frac{2 \pi}{N} k n \quad, k=0,1,2, \ldots, N-1} \\
S_{\hat{x} \hat{x}}(k)=\frac{1}{N T}|\hat{X}(k)|^{2} \ldots \ldots \ldots \ldots . . . . . . .
\end{array}
$$

\section{Cross-correlation function}

For cross-correlation function (CCF), the signals are first bandpass filtered into three frequency ranges, the VLF range which consisted of $0 \mathrm{~Hz}$ to $0.07 \mathrm{~Hz}$, the LF range which consisted of $0.07 \mathrm{~Hz}$ to 0.15 $\mathrm{Hz}$, and the $\mathrm{HF}$ range, which is the same as the HF range for when calculating PSD. In this study, a third-order digital band pass Type I Chebyshev filter was applied to both the MBP and MCBFV signals, with the pass band ripple error limited to $0.1 \mathrm{~dB}$. Let the pass band filtered signals of MBP and MCBFV be $\hat{\mathrm{f}}(\mathrm{n})$ and $\hat{\mathrm{g}}(\mathrm{n})$ respectively, then the CCF can be calculated as shown in (5).

$$
\begin{gathered}
C C F_{i}(k)=\frac{R_{\hat{f} \hat{g}}^{i}(k)}{\left[R_{\hat{f} \hat{g}}^{i}(0) R_{\hat{f} \hat{g}}^{i}(0)\right]^{\frac{1}{2}}}, k=0, \pm 1, \pm 2, \ldots, i=1,2, \cdots, N-W+ \\
R_{\hat{f} \hat{g}}^{i}(k)=\left\{\begin{array}{c}
\frac{1}{W} \sum_{j=i}^{i+W} \hat{f}(j) \hat{g}(j+k), k=0,1,2, \cdots \\
\frac{1}{W} \sum_{j=i}^{i+W} \hat{f}(j-k) \hat{g}(j), k=0,-1,-2, \ldots
\end{array}\right.
\end{gathered}
$$

Where $R_{\hat{f g}}^{i}(k)$ is the estimate of the cross-covariance in the $i^{\text {th }}$ window defined above in (6), $\mathrm{N}$ is the total number of cardiac cycles, $\mathrm{W}$ is the window width, and $\mathbf{k}$ is the time lag. The mean CCF patterns were obtained for each subject.

\section{Statistical tests}

All statistical tests were calculated using R, where ANOVA was used to test for significant differences between the control group and the PD group in each analysis method.

\section{RESULTS}

\section{Mean analysis results}

Both the MBP and MCBFV signals were averaged to find the mean of each signal to see whether or not a significant difference existed for when the subjects were at rest, tilt, or hyper. The results are shown in table 1.

From the results shown in table 1, ANOVA showed no significant differences between the subject groups in any of the stimulations or any of the signals.

\section{Power spectral density analysis results}

PSD was done for all subjects during each of the different actions. The frequency bands here should reflect the heart rate variability frequency bands, which show the balance in the autonomic nervous system.

\section{Cross-correlation function analysis results}

CCF was also done for all subjects during each of the different actions. The mean and standard deviation of the CCFs was found for all time indices; then the highest peak was taken and used to denote the greatest time lag between MBP and MCBFV. The MAX CCF value is the value of the highest peak of the mean CCF, and the CCF SD value is the corresponding SD value, and the CCF INDEX is the time lag of the highest peak of the mean CCF.

Table 1: Results of mean analysis

\begin{tabular}{lllllll}
\hline Type & MBP REST & MCBFV REST & MBP TILT & MCBFV TILT & MBP HV \\
\hline Control & $88.19 \pm 8.47$ & $38.29 \pm 11.06$ & $94.80 \pm 11.61$ & $36.76 \pm 10.80$ & $91.82 \pm 9.10$ & $26.66 \pm 9.64$ \\
PD & $85.93 \pm 15.10$ & $34.95 \pm 12.22$ & $88.08 \pm 17.86$ & $33.03 \pm 12.53$ & $93.64 \pm 16.70$ & $31.77 \pm 11.02$ \\
\hline
\end{tabular}

Table 2: Results of power spectral density analysis during rest

\begin{tabular}{lllllll}
\hline Type & MBP VLF & MCBFV VLF & MBP LF & MCBFV LF & MBP HF & MCBFV HF \\
\hline Control & $0.33 \pm 0.10$ & $0.28 \pm 0.14$ & $0.26 \pm 0.16$ & $0.22 \pm 0.15$ & $0.26 \pm 0.180$ & $0.19 \pm 0.23$ \\
PD & $0.233 \pm 0.121$ & $0.31 \pm 0.11$ & $0.27 \pm 0.19$ & $0.12 \pm 0.10$ & $0.28 \pm 0.20$ & $0.13 \pm 0.16$ \\
\hline
\end{tabular}

Table 3: Results of power spectral density analysis during tilt

\begin{tabular}{llllll}
\hline Type & MBP VLF & MCBFV VLF & MBP LF & MCBFV LF & MBP HF \\
\hline Control & $0.31 \pm 0.15$ & $0.23 \pm 0.08$ & $0.23 \pm 0.15$ & $0.19 \pm 0.18$ & $0.19 \pm 0.23$ \\
PD & $0.25 \pm 0.08$ & $0.30 \pm 0.10$ & $0.19 \pm 0.12$ & $0.22 \pm 0.10$ & $0.18 \pm 0.17$ \\
\hline
\end{tabular}

Table 4: Results of power spectral density analysis during hyperventilation

\begin{tabular}{llllll}
\hline Type & MBP VLF & MCBFV VLF & MBP LF & MCBFV LF* & MBP HF \\
\hline Control & $0.16 \pm 0.08$ & $0.27 \pm 0.14$ & $0.13 \pm 0.10$ & $0.25 \pm 0.12$ & $0.26 \pm 0.25$ \\
PD & $0.15 \pm 0.10$ & $0.22 \pm 0.13$ & $0.16 \pm 0.11$ & $0.16 \pm 0.08$ & $0.15 \pm 0.130$ \\
\hline
\end{tabular}

There are no statistically significant differences between groups in table 2 and table 3 . In table 4 , MCBFV LF has been marked with a $*$ to denote that the difference between groups is significant at $p \leq 0.05$. 
Table 5: Results of cross-correlation function analysis during rest

\begin{tabular}{llll}
\hline Type & VLF MAX CCF & VLF CCF SD & VLF CCF INDEX \\
\hline Control & $0.44 \pm 0.22$ & $0.30 \pm 0.11$ & $-2.28 \pm 5.23$ \\
PD & $0.22 \pm 0.32$ & $0.323 \pm 0.09$ & $-4.24 \pm 6.94$ \\
Type & LF-MAX CCF & LF CCF SD & LF CCF INDEX \\
Control & $0.47 \pm 0.16$ & $0.27 \pm 0.06$ & $-1.64 \pm 0.59$ \\
PD & $0.34 \pm 0.17$ & $0.30 \pm 0.09$ & $-1.42 \pm 2.43$ \\
Type & HF MAX CCF & HF CCF SD & HF CCF INDEX \\
Control & $0.21 \pm 0.11$ & $0.19 \pm 0.04$ & $-0.39 \pm 0.92$ \\
PD & $0.26 \pm 0.17$ & $0.19 \pm 0.06$ & $-0.19 \pm 0.66$ \\
\hline
\end{tabular}

Table 6: Results of cross-correlation function analysis during tilt

\begin{tabular}{llll}
\hline Type & VLF MAX CCF** & VLF CCF SD* & VLF CCF INDEX*** \\
\hline Control & $0.51 \pm 0.19$ & $0.30 \pm 0.09$ & $-1.30 \pm 2.60$ \\
PD & $0.24 \pm 0.27$ & $0.40 \pm 0.10$ & $-4.51 \pm 2.86$ \\
Type & LF-MAX CCF & LF CCF SD & LF CCF INDEX \\
Control & $0.55 \pm 0.20$ & $0.22 \pm 0.09$ & $-1.53 \pm 1.07$ \\
PD & $0.51 \pm 0.26$ & $0.25 \pm 0.13$ & $-1.21 \pm 2.00$ \\
Type & HF MAX CCF & HF CCF SD & HF CCF INDEX \\
Control & $0.28 \pm 0.12$ & $0.21 \pm 0.06$ & $-0.30 \pm 0.74$ \\
PD & $0.35 \pm 0.15$ & $0.20 \pm 0.06$ & $-0.08 \pm 0.55$ \\
\hline
\end{tabular}

Table 7: Results of cross-correlation function analysis during hyperventilation

\begin{tabular}{llll}
\hline Type & VLF MAX CCF & & VLF CCF INDEX \\
\hline Control & $0.06 \pm 0.32$ & VLF CCF SD & $-3.11 \pm 6.97$ \\
PD & $0.39 \pm 0.32$ & $0.39 \pm 0.13$ & $-4.18 \pm 3.19$ \\
Type & LF MAX CCF* & $0.34 \pm 0.15$ & LF CCF INDEX \\
Control & $0.27 \pm 0.09$ & LF CCF SD & $-3.09 \pm 1.76$ \\
PD & $0.41 \pm 0.15$ & $0.35 \pm 0.10$ & $-1.93 \pm 0.83$ \\
Type & HF MAX CCF & $0.29 \pm 0.13$ & HF CCF INDEX \\
Control & $0.18 \pm 0.07$ & HF CCF SD & $0.06 \pm 1.33$ \\
PD & $0.25 \pm 0.16$ & $0.20 \pm 0.06$ & $0.21 \pm 0.82$ \\
\hline
\end{tabular}

There are no statistically significant differences between groups in table 5. In Tables 6, VLF CCF SD and in table 7 VLF MAX CCF and LF-MAX CCF has been marked with $\mathrm{a}^{*}$ to denote that the difference between groups is significant at $p \leq 0.05$, and in table 6 , VLF MAX CCF has been marked with a ** to denote that the difference between groups is significant at $p \leq 0.01$, while in table 6 , VLF CCF INDEX has been marked with a $* * *$ to denote that the difference between groups is significant at $p \leq 0.001$.

\section{DISCUSSION}

It is important to note that there were no significant differences that can be seen between the control and PD groups while the subjects are at rest. This means that while lying down in a supine position, a PD patient's ANS and CA may operate like a healthy person's. However, during tilt, there is a significant difference between the VLF band during the CCF analysis between the PD and controls. Here, the controls showed a higher correlation and a smaller time lag, while the PD patients showed a lower correlation and a higher time lag. While it is still largely unknown what the VLF band may tell us, it is still important to note for the future that there is a very significant difference between the groups.

The most important discovery of this study is the significant differences found when the subjects are hyperventilating. This creates a hypocapnic condition, where the healthy controls are meant to have a faster and better CA reaction [7]. From the LF band of the CCF analysis, it can be seen that this is the case for the healthy controls, as the time lag index is much slower during hyperventilation, as well as the max CCF value is lower during hyperventilation. However, it would appear that for PD patients, the CA is not improved as much as controls during hypocapnia, thus giving the impression that CA is impaired, and can be seen during hypocapnic conditions, but not normal conditions.

\section{CONCLUSION}

The findings in this study agree with previous studies that CA may be affected for PD patients, and also shows why some previous studies have found the matter to be inconclusive. However, an important fact found in this study was that the impairment can be more clearly seen while the subjects are under hyperventilation, during a hypocapnic condition where CA is improved in the healthy controls, while they are not improved for the PD patients. Since CA is stated to be controlled by three mechanisms (metabolic, myogenic, and neurogenic), it is perhaps the metabolic regulation is damaged, as the change in the concentration of oxygen and carbon dioxide is the one that seems to be affected, rather than the neurogenic pathway that other autonomic disorders seem to show. More research into CCF and PSD analysis with the different pathways with a larger group of subjects should be a goal for future researches in this field in order to clarify the results.

\section{ACKNOWLEDGMENT}

The authors would like to thank the Ministry of Science and Technology (MoST) of Taiwan, R. O. C. for supporting and providing the funding for this research under contract number MOST102-2221-E-035-004-MY3.

\section{CONFLICTS OF INTERESTS}

All authors have none to declare.

\section{REFERENCES}

1. Chaudhuri KR, Healy DG, Schapira AH. Non-motor symptoms of Parkinson's disease: diagnosis and management. Lancet Neurol 2006;5:235-45.

2. Wakabayashi K, Takahashi H. Neuropathology of autonomic nervous system in Parkinson's disease. Eur Neurol 1997;38(Suppl 2):2-7. 
3. Senard JM, Rai S, Lapeyre-Mestre M, Brefel C, Rascol O, Rascol $A$, et al. Prevalence of orthostatic hypotension in Parkinson's disease. J Neurol Neurosurg Psychiatry 1997;63:584-9.

4. Lagi A, Bacalli S, Cencetti S, Paggetti C, Colzi L. Cerebral autoregulation in orthostatic hypotension. A transcranial Doppler study. Stroke 1994;25:1771-5.

5. Vokatch N, Grötzsch H, Mermillod B, Burkhard PR, Sztajzel R. Is cerebral autoregulation impaired in Parkinson's disease? A transcranial Doppler study. J Neurol Sci 2007;254:49-53.

6. Haunton VJ. Is dynamic cerebral autoregulation impaired in idiopathic Parkinson's disease? Doctoral dissertation, Department of Cardiovascular Sciences, University of Leicester, Leicester, England; 2014.

7. Aaslid R, Lindegaard KF, Sorteberg W, Nornes H. Cerebral autoregulation dynamics in humans. Stroke 1989;20:45-52.

8. Lin SL, Liao AYC, Yeh SJ. The cardiovascular and respiratory responses to $\mathrm{CO}_{2}$ under hyperventilation and posture change in Parkinson's patients. J Med Bioeng 2015; 4:350-6.

9. Lin SL, Liao AYC, Yeh SJ, Lin JY. The analysis of cardio-respiratory signals and cerebral autoregulation based on $\mathrm{CO} 2$ reactivity with healthy subjects and Parkinson's patients. Technol Health Care 2015;24 Suppl 1:195-203.

10. Chiu CC, Yeh SJ. Assessment of cerebral autoregulation using time-domain cross-correlation analysis. Comput Biol Med 2001;31: 471-80.

11. Chiu CC, Yeh SJ, Liau BY. Assessment of cerebral autoregulation dynamics in diabetics using time-domain cross-correlation analysis. J Med Biol Eng 2005;25:53-9.

12. Chiu CC, Yeh SJ, Hu YH, Liao KYK. SVM classification for diabetics with various degrees of autonomic neuropathy based on cross-correlation features. J Med Biol Eng 2014;34:495-500.

13. Low PA. Composite autonomic scoring scale for laboratory quantification of generalized autonomic failure. Mayo Clin Proc 1993;68:748-52.

\section{How to cite this article}

- $\quad$ Chuang Chien Chiu, Ken Ying-Kai Liao, Shoou Jeng Yeh. Linear assessment of cerebral autoregulation in idiopathic Parkinson's disease. Int J Pharm Pharm Sci 2016;8 Suppl 2:14-17. 